\title{
Introduction to the special issue on evolutionary intelligence in games
}

\author{
J. J. Merelo $\cdot$ Paolo Burelli
}

Received: 6 January 2014 / Accepted: 7 January 2014/Published online: 24 January 2014

(C) Springer-Verlag Berlin Heidelberg 2014

\section{Presentation}

This issue includes three papers selected from those presented to the EvoGames track within the EvoStar 2013 conference (Esparcia-Alcázar 2013). All authors with a paper accepted in that conference were invited and then an open call was made to anybody working in the topic of evolutionary intelligence in games. After the authors submitted an extended version a selection was made and these three papers were accepted after two rounds of revision.

The three papers represent a wide range of games and puzzles: a relatively recent trading-card game, Dominion (Mahlmann et al. 2012), which was created by Donald Vacarino and published by Rio Grande Games in 2008, a classic platform game, Super Mario (Togelius et al. 2009) and a board game, MasterMind, which is actually a puzzle proposed initially in the $70 \mathrm{~s}$ by a telecommunication engineer and whose first solution was proposed by Donald Knuth a few years later (Knuth 1976-77).

The first paper by Ransom Winder (2014) is the one that tries to find a winning deck of ten cards for the Dominion game; in fact, the problem that is tackled is to try and predict the game states to make decisions about the next

\section{J. J. Merelo $(\square)$}

Departamento de Arquitectura y Tecnología de Computadores, Universidad de Granada, Granada, Spain

e-mail: jmerelo@geneura.ugr.es

P. Burelli

Aalborg University, Aalborg, Denmark move. Neural networks are applied to the state of the game when the player has to make any decision, and these neural networks have to be trained so that the outcome of these decisions is as favorable to the player as possible. Several methods have been tried, and an evolutionary-algorithm based game bot is the only one that obtains an edge over classical strategies.

The second paper also combines evolutionary algorithms with other algorithms, in this case Finite State Automata, to evolve in this case strategies for advancing a Super Mario avatar in platforms of increasing difficulty (Mora et al. 2014). This game has been traditionally used for computational intelligence competitions; FSM are also traditionally used to drive game bots in strategy and first person shooter games. But designing them can be tricky, and an evolutionary algorithm that designs the transitions between states is successfully used in this paper for a Mario that is able to obtain good results up to a high level; besides, a study is made on the population needed to obtain good results in each level.

The final paper is more purely evolutionary (MaestroMontojo et al. 2014) in the sense that it does not combine evolutionary techniques with others in order to solver the MasterMind game, a puzzle in which a secret combination must be found with the help of hits given as response to every move made by a player. The paper approaches this problem from two different angles: first, evolving populations of combinations scores according to the hints made and then using genetic programming to evolve good scoring methods for these combinations that have, traditionally, been chosen heuristically.

Summing up, this special issue includes a diverse set of papers that use evolutionary algorithms to find solutions to a wide range of games combining them with other techniques such as neural nets or finite state automata. 


\section{References}

Esparcia-Alcázar AI (ed) (2013) Applications of evolutionary computation-16th European conference, EvoApplications 2013, Vienna, Austria, April 3-5, 2013. In: Proceedings, Lecture notes in computer science, vol 7835, Springer, Berlin

Knuth DE (1976-77) The computer as master mind. J Recreat Math 9(1):1-6

Maestro-Montojo J, Salcedo-Sanz S, Merelo JJ (2014) New solver and optimal anticipation strategies design based on evolutionary computation for the game of MasterMind. Evol Intell. doi:10. 1007/s12065-013-0099-6

Mahlmann T, Togelius J, Yannakakis GN (2012) Evolving card sets towards balancing dominion. In: Evolutionary computation (CEC), 2012 IEEE congress on, IEEE, pp 1-8
Mora A, Merelo J, García-Sánchez P, Castillo P, Rodríguez-Domingo M, Hidalgo-Bermúdez R (2014) Creating autonomous agents for Super Mariogame with evolutionary FSMs. Evol Intell. doi:10. 1007/s12065-014-0105-7

Togelius J, Karakovskiy S, Koutník J, Schmidhuber J (2009) Super Mario evolution. In: Computational intelligence and games, 2009. CIG 2009. IEEE symposium on, IEEE, pp 156-161

Winder RK (2014) Methods for approximating value functions for the Dominion card game. Evol Intell pp 1-10, http://www.scopus. com, article in Press 